\title{
Karakteristik Konikoida
}

\author{
Sahlan Sidjara $^{1 *}$, Muhammad Abdy $^{2}$ \\ ${ }^{1,2}$ Jurusan Matematika, FMIPA, Universitas Negeri Makassar \\ *corresponding author email: sahlansidjara@unm.ac.id
}

\begin{abstract}
Abstrak
Pada geometri bidang khususnya pada kasus irisan kerucut terdapat beberapa bentuk yang dapat diperoleh dari irisan kerucut diantaranya: Lingkaran, Elips, Hiperbola dan Parabola. Selanjutnya, bentuk-bentuk tersebut pada geometri ruang disebut sebagai konikoida yang terdiri dari: bola, elipsoida, kerucut eliptik, hiperboloida daun satu, hiperboloida daun dua, paraboloida eliptik, paraboloida hiperboloida,silinder hiperbolik dan silinder parabolik. Tulisan ini membahas mengenai karakteristik dari konikoida berdasakan kerucut arah dan pusat konikoida.
\end{abstract}

Kata Kunci: konikoida, kerucut arah dan pusat konikoida.

\section{The Characteristics Of Conicoid}

\begin{abstract}
On the plane geometry study especially in the case of conic slices, there are several forms from conic slices for example: cirlce, ellips, hypernolic and parabolic.However, these forms on the space of geometry known as conicoid consisting of: sphare, ellipsoid, cone elliptic, one leaves hyperboloid, two leaves hyperbolid, elliptic parabolod, hyperboloid parabolic, hyperboloid cylinder, elliptic cylinder and parabolic cylinder. This paper discusses about the characteristics of conicoid besed on its center and cone direction.
\end{abstract}

Keywords:: conicoid, center conicoid and cone direction.

\section{Pendahuluan}

Kata "geometri" berasal dari bahasa yunani (greek) yang berarti "ukuran bumi". Maksudnya mencakup mengukur segala sesuatu yang ada di bumi. Geometri kuno sebagian dimulai dari pengukuran praktis yang diperlukan untuk pertanian orang-orang Babylonia dan Mesir. Kemudian geometri orang Mesir dan Babylonia ini diperluas untuk perhitungan panjang ruas garis, luas dan volume. Berdasarkan ruang lingkup atau bidang kajiannya, geometri terdiri atas beberapa kelompok salah satu diantaranya adalah geometri bidang (dimensi dua) dan geometri ruang (dimensi tiga). Dalam geometri bidang (dimensi dua), terdapat penjelasan mengenai konik (irisan kerucut) [1]. Menurut [2], Irisan antara kerucut dengan bidang rata (dimensi dua) meliputi: lingkaran, elips, hiperbola dan parabola, untuk lebih jelasnya, perhatikan gambar 1.

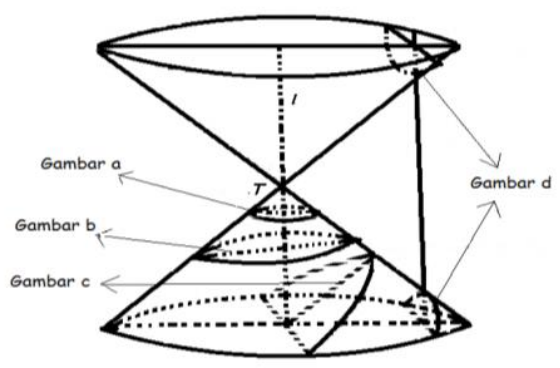

Gambar 1. Irisan Kerucut

1) Lingkaran diperoleh dengan memotong bagian dari selimut kerucut dengan sebuah bidang rata yang tegak lurus terhadap sumbu kerucut $(l)$ tetapi tidak melalui titik T (gambar a).

2) Elips diperoleh dengan memotong bagian dari selimut kerucut dengan bidang rata dan tidak tegak lurus terhadap sumbu kerucut ( $l$ ) (gambar b). 
3) Parabola diperoleh dengan memotong kerucut dengan bidang rata yang sejajar dengan pelukis kerucut (gambar c).

4) Hiperbola diperoleh dengan memotong selimut kerucut (selimut bagian atas dan bawah) dengan bidang rata yang sejajar dengan sumbu kerucut $(l)$ (gambar d).

Lingkaran, hiperbola, parabola dan elips adalah bangun-bangun pada geometri bidang (dimensi dua). Bola, hiperboloida, paraboloida dan elipsoida adalah bangun-bangun pada geometri ruang (dimensi tiga) dan merupakan bentuk-bentuk dari konikoida [1].

Menurut [3], menyatakan bahwa secara umum persamaan umum konikoida berbentuk $f(x, y, z)=$ $a_{11} x^{2}+a_{22} y^{2}+a_{33} z^{2}+2 a_{12} x y+2 a_{13} x z+2 a_{23} y z+2 a_{14} x+2 a_{24} y+2 a_{34} z+a_{44}=0$ dengan paling sedikit $a_{11}, a_{22}, a_{33}, a_{12}, a_{13}$, dan $a_{23}$ tidak sama dengan nol. Kerucut arah (KA) konikoida diperoleh dengan menghapus bagian linear dan bagian konstanta dari konikoida yaitu:

$$
\mathrm{KA}: a_{11} x^{2}+a_{22} y^{2}+a_{33} z^{2}+2 a_{12} x y+2 a_{13} x z+2 a_{23} y z=0
$$

atau secara matriks persamaan konikoida tersebut dapat ditulis:

Atau:

$$
\left[\begin{array}{lll}
x & y & z
\end{array}\right]\left[\begin{array}{lll}
a_{11} & a_{12} & a_{13} \\
a_{12} & a_{22} & a_{23} \\
a_{13} & a_{23} & a_{33}
\end{array}\right]\left[\begin{array}{l}
x \\
y \\
z
\end{array}\right]+2\left[\begin{array}{lll}
a_{14} & a_{24} & a_{34}
\end{array}\right]\left[\begin{array}{l}
x \\
y \\
z
\end{array}\right]+\left[a_{44}\right]=0
$$

$V^{T} A V+2 B^{T} V+C=0$ dengan

$A=\left[\begin{array}{lll}a_{11} & a_{12} & a_{13} \\ a_{12} & a_{22} & a_{23} \\ a_{13} & a_{23} & a_{33}\end{array}\right], V=\left[\begin{array}{l}x \\ y \\ z\end{array}\right], B=\left[\begin{array}{l}a_{14} \\ a_{24} \\ a_{34}\end{array}\right]$ dan $C=\left[a_{44}\right]$

$V^{T} A V$ disebut sebagai bagian homogen kuadratis.

$2 B^{T} V$ disebut bagian linear dan

$C$ disebut konstanta dari konikoida.

Jenis-jenis konikoida antara lain bola, elipsoida, kerucut eliptik,hiperboloida daun satu, hiperboloida daun dua, paraboloida eliptik, paraboloida hiperboloida,silinder hiperbolik, silinder parabolik dapat dilihat di [1] dan penjelasan mengenai rank dari suatu matriks dapat dilihat di [3].

\section{Pembahasan}

\subsection{Kerucut Arah}

Berikut ini merupakan penggolongan konikoida berdasarkan kerucut arah.

Teorema 2.1. Berdasarkan persamaan umum konikoida: $\quad f(x, y, z)=a_{11} x^{2}+a_{22} y^{2}+a_{33} z^{2}+$ $2 a_{12} x y+2 a_{13} x z+2 a_{23} y z+2 a_{14} x+2 a_{24} y+2 a_{34} z+a_{44}=0$, diiris dengan $\mathrm{z}=1$ maka diperoleh persamaan

$$
\begin{array}{r}
f(x, y)=a_{11} x^{2}+a_{22} y^{2}+2 a_{12} x y+2 a_{13} x+2 a_{23} y+a_{33}=0 \\
\text { Jika } D=\left|\begin{array}{ll}
a_{11} & a_{12} \\
a_{12} & a_{22}
\end{array}\right|, H=\left|\begin{array}{lll}
a_{11} & a_{12} & a_{13} \\
a_{12} & a_{22} & a_{23} \\
a_{13} & a_{23} & a_{33}
\end{array}\right| \text { dan } S=a_{11}+a_{22}
\end{array}
$$

Maka

Untuk $H \neq 0$, diperoleh irisan kerucut arah dengan bidang $z=1$ yaitu kerucut sejati yang terdiri dari:

(1) Jika $D>0, \frac{S}{H}<0$ maka diperoleh elips nyata.

(2) Jika $D>0, \frac{S}{H}>0$ maka diperoleh elips khayal

(3) Jika $D<0$ maka diperoleh hiperbola (selalu nyata).

(4) Jika $D=0$ maka diperoleh parabola (selalu nyata).

Untuk $H=0$, diperoleh irisan kerucut arah dengan bidang $z=1$ berubah corak menjadi sepasang garis lurus yang terdiri dari:

(1) Jika $D>0$ maka diperoleh sepasang garis khayal.

(2) Jika $D<0$ maka diperoleh sepasang garis nyata berpotongan

(3) Jika $D=0$ maka diperoleh sepasang garis sejajar atau berhimpit. 


\section{Bukti}

Berdasarkan persamaan umum konikoida:

$$
\begin{aligned}
f(x, y, z)= & a_{11} x^{2}+a_{22} y^{2}+a_{33} z^{2}+2 a_{12} x y+2 a_{13} x z+2 a_{23} y z+2 a_{14} x+ \\
& 2 a_{24} y+2 a_{34} z+a_{44}=0 .
\end{aligned}
$$

Diiris dengan bidang $\mathrm{z}=1$ maka diperoleh:

$$
\begin{aligned}
& f(x, y)=a_{11} x^{2}+a_{22} y^{2}+a_{33}+2 a_{12} x y+2 a_{13} x+2 a_{23} y+2 a_{14} x+2 a_{24} y+2 a_{34}+a_{14}=0 . \\
& \Rightarrow \quad f(x, y)=a_{11} x^{2}+a_{22} y^{2}+2 a_{12} x y+\left(2 a_{13} x+2 a_{14} x\right)+\left(2 a_{23} y+\right. \\
& \left.\quad 2 a_{24} y\right)+\left(2 a_{34}++a_{33}+a_{14}\right)=0 . \\
& \Rightarrow \quad f(x, y)=a_{11} x^{2}+a_{22} y^{2}+2 a_{12} x y+\left(2 a_{13}+2 a_{14}\right) x+\left(2 a_{23}+2 a_{24}\right) y+\left(2 a_{34}+a_{33}+\right. \\
& \left.\quad a_{14}\right)=0 .
\end{aligned}
$$

\section{Untuk $\boldsymbol{H} \neq \mathbf{0}$}

\section{Bukti (1)}

Diketahui jika $H \neq 0, D>0$ dan $S / H<0$ irisan merupakan elips nyata

Ambil $a_{11}=-a, a_{22}=-b, a_{33}=c$ dan $a_{12}=a_{13}=a_{23}=0$ dengan $(a, b, c \in$ bilangan positif $)$ sehingga:

$$
\begin{aligned}
& H=\left|\begin{array}{lll}
a_{11} & a_{12} & a_{13} \\
a_{12} & a_{22} & a_{23} \\
a_{13} & a_{23} & a_{33}
\end{array}\right|=\left|\begin{array}{ccc}
-a & 0 & 0 \\
0 & -b & 0 \\
0 & 0 & c
\end{array}\right|=a b c \neq 0 \\
& D=\left|\begin{array}{ll}
a_{11} & a_{12} \\
a_{12} & a_{22}
\end{array}\right|=\left|\begin{array}{cc}
-a & 0 \\
0 \quad-b
\end{array}\right|=a b>0 \\
& S=a_{11}+a_{22}=-a-b=-(a+b) \\
& \frac{S}{H}=\frac{-(a+b)}{a b c}<0
\end{aligned}
$$

Maka berdasarkan persamaan (1) diperoleh:

$$
\begin{aligned}
& a_{11} x^{2}+a_{22} y^{2}+2 a_{12} x y+2 a_{13} x+2 a_{23} y+a_{33}=0 \\
& \Rightarrow-a x^{2}-b y^{2}+c=0 \\
& \Rightarrow-a x^{2}-b y^{2}=-c \\
& \Rightarrow-\left(a x^{2}+b y^{2}\right)=-c \\
& \Rightarrow a x^{2}+b y^{2}=c \\
& \Rightarrow \frac{a}{c} x^{2}+\frac{b}{c} y^{2}=1 \\
& \Rightarrow \frac{x^{2}}{\frac{c}{a}}+\frac{y^{2}}{\frac{c}{b}}=1 \\
& \Rightarrow \frac{x^{2}}{\left(\sqrt{\frac{c}{a}}\right)^{2}}+\frac{y^{2}}{\left(\sqrt{\frac{c}{b}}\right)^{2}}=1 \text { (merupakan elips nyata). }
\end{aligned}
$$

\section{Bukti (2)}

Diketahui jika $H \neq 0, D>0$ dan $S / H>0 \Rightarrow$ irisan merupakan elips khayal

Ambil $a_{11}=-a, a_{22}=-b, a_{33}=-c$ dan $a_{12}=a_{13}=a_{23}=0$ dengan $\quad(a, b, c \in$ bilangan positif) sehingga:

$H=\left|\begin{array}{lll}a_{11} & a_{12} & a_{13} \\ a_{12} & a_{22} & a_{23} \\ a_{13} & a_{23} & a_{33}\end{array}\right|=\left|\begin{array}{ccc}-a & 0 & 0 \\ 0 & -b & 0 \\ 0 & 0 & -c\end{array}\right|=-a b c \neq 0$
$D=\left|\begin{array}{ll}a_{11} & a_{12} \\ a_{12} & a_{22}\end{array}\right|=\left|\begin{array}{cc}-a & 0 \\ 0 & -b\end{array}\right|=a b>0$
$S=a_{11}+a_{22}=-a-b=-(a+b)$
$\frac{S}{H}=\frac{-(a+b)}{-a b c}>0$

Maka berdasarkan persamaan (1) diperoleh:

$$
\begin{aligned}
& a_{11} x^{2}+a_{22} y^{2}+2 a_{12} x y+2 a_{13} x+2 a_{23} y+a_{33}=0 \\
& \Rightarrow \quad-a x^{2}-b y^{2}-c=0 \\
& \Rightarrow \quad \frac{a}{c} x^{2}+\frac{b}{c} y^{2}=-1 \\
& \Rightarrow \quad \frac{x^{2}}{\frac{c}{a}}+\frac{y^{2}}{\frac{c}{b}}=-1
\end{aligned}
$$


$\Rightarrow \frac{x^{2}}{\left(\sqrt{\frac{c}{a}}\right)^{2}}+\frac{y^{2}}{\left(\sqrt{\frac{c}{b}}\right)^{2}}=-1$ (merupakan elips khayal).

Bukti (3)

Diketahui jika $H \neq 0, D<0 \Rightarrow$ irisan merupakan hiperbola

Ambil $a_{11}=a, a_{22}=-b, a_{33}=c$ dan $a_{12}=a_{13}=a_{23}=0$ dengan $(a, b, c \in$ bilangan positif) sehingga:

$H=\left|\begin{array}{lll}a_{11} & a_{12} & a_{13} \\ a_{12} & a_{22} & a_{23} \\ a_{13} & a_{23} & a_{33}\end{array}\right|=\left|\begin{array}{ccc}a & 0 & 0 \\ 0 & -b & 0 \\ 0 & 0 & c\end{array}\right|=-a b c \neq 0$

$D=\left|\begin{array}{ll}a_{11} & a_{12} \\ a_{12} & a_{22}\end{array}\right|=\left|\begin{array}{cc}a & 0 \\ 0 & -b\end{array}\right|=-a b<0$

Maka berdasarkan persamaan (1) diperoleh:

$a_{11} x^{2}+a_{22} y^{2}+2 a_{12} x y+2 a_{13} x+2 a_{23} y+a_{33}=0$

$\Rightarrow a x^{2}-b y^{2}-c=0$

$\Rightarrow \quad \frac{a}{c} x^{2}-\frac{b}{c} y^{2}=1$

$\Rightarrow \quad \frac{x^{2}}{\frac{c}{a}}-\frac{y^{2}}{\frac{c}{b}}=1$

$\Rightarrow \quad \frac{x^{2}}{\left(\sqrt{\frac{c}{a}}\right)^{2}}-\frac{y^{2}}{\left(\sqrt{\frac{c}{b}}\right)^{2}}=1$ (merupakan hiperbola).

Bukti (4)

Diketahui jika $H \neq 0, D=0 \Rightarrow$ irisan merupakan parabola

Ambil $a_{11}=a, a_{23}=-b$ dan $a_{12}=a_{13}=a_{22}=a_{33}=0$ dengan $(a, b, \in$ bilangan positif) sehingga:

$H=\left|\begin{array}{lll}a_{11} & a_{12} & a_{13} \\ a_{12} & a_{22} & a_{23} \\ a_{13} & a_{23} & a_{33}\end{array}\right|=\left|\begin{array}{ccc}a & 0 & 0 \\ 0 & 0 & -b \\ 0 & -b & 0\end{array}\right|=-a b^{2} \neq 0$

$D=\left|\begin{array}{ll}a_{11} & a_{12} \\ a_{12} & a_{22}\end{array}\right|=\left|\begin{array}{ll}a & 0 \\ 0 & o\end{array}\right|=0$

Maka berdasarkan persamaan (1) diperoleh:

$a_{11} x^{2}+a_{22} y^{2}+2 a_{12} x y+2 a_{13} x+2 a_{23} y+a_{33}=0$

$\Rightarrow \quad-a x^{2}-2 b y=0$

$\Rightarrow \quad-2 b y=-a x^{2}$

$\Rightarrow \quad 2 b y=a x^{2}$

$\Rightarrow y=\frac{a}{2 b} x^{2}$ (merupakan parabola).

\section{Untuk $\boldsymbol{H} \neq \mathbf{0}$}

\section{Bukti (1)}

Diketahui jika $H=0, D>0 \Rightarrow$ irisan merupakan sepasang garis khayal.

Ambil $a_{11}=a_{22}=a$, dan $a_{12}=a_{13}=a_{23}=a_{33}=0$ dengan $(a, \in$ bilangan positif $)$ sehingga:

$H=\left|\begin{array}{lll}a_{11} & a_{12} & a_{13} \\ a_{12} & a_{22} & a_{23} \\ a_{13} & a_{23} & a_{33}\end{array}\right|=\left|\begin{array}{lll}a & 0 & 0 \\ 0 & a & 0 \\ 0 & 0 & 0\end{array}\right|=0$

$D=\left|\begin{array}{ll}a_{11} & a_{12} \\ a_{12} & a_{22}\end{array}\right|=\left|\begin{array}{cc}a & 0 \\ 0 & a\end{array}\right|=a^{2}>0$

Maka berdasarkan persamaan (1) diperoleh:

$a_{11} x^{2}+a_{22} y^{2}+2 a_{12} x y+2 a_{13} x+2 a_{23} y+a_{33}=0$

$\Rightarrow a x^{2}+a y^{2}=0$

$\Rightarrow a\left(x^{2}+y^{2}\right)=0$

$\Rightarrow x^{2}+y^{2}=0$

$\Rightarrow \quad x^{2}=-y^{2}$

$\Rightarrow \quad x= \pm \sqrt{-y^{2}}$ (merupakan sepasang garis khayal)

\section{Bukti (3)}

Diketahui jika $H=0, D=0 \Rightarrow$ irisan merupakan sepasang garis berhimpit

Ambil $a_{11}=a_{22}=a_{12}=a$, dan $a_{13}=a_{23}=a_{33}=0$ dengan $(a, \in$ bilangan positif) sehingga: 
$H=\left|\begin{array}{lll}a_{11} & a_{12} & a_{13} \\ a_{12} & a_{22} & a_{23} \\ a_{13} & a_{23} & a_{33}\end{array}\right|=\left|\begin{array}{lll}a & a & 0 \\ a & a & 0 \\ 0 & 0 & 0\end{array}\right|=0$
$D=\left|\begin{array}{ll}a_{11} & a_{12} \\ a_{12} & a_{22}\end{array}\right|=\left|\begin{array}{ll}a & a \\ a & a\end{array}\right|=0$

Maka berdasarkan persamaan (1) diperoleh:

$a_{11} x^{2}+a_{22} y^{2}+2 a_{12} x y+2 a_{13} x+2 a_{23} y+a_{33}=0$

$\Rightarrow a x^{2}+a y^{2}+2 a x y=0$

$\Rightarrow a\left(x^{2}+y^{2}+2 x y\right)=0$

$\Rightarrow \quad x^{2}+y^{2}+2 x y=0$

$\Rightarrow \quad(x+y)(x+y)=0$ (merupakan sepasang garis berhimpit).

\subsection{Pusat Konikoida}

Persamaan umum konikoida $f(x, y, z)=a_{11} x^{2}+a_{22} y^{2}+a_{33} z^{2}+2 a_{12} x y+2 a_{13} x z+$ $2 a_{23} y z+2 a_{14} x+2 a_{24} y+2 a_{34} z+a_{44}=0$ dengan pusat $P\left(x_{1}, y_{1}, z_{1}\right)$

Definisi 2.2. [6] Persamaan pusat konikoida $f(x, y, z)$ dengan pusat $P\left(x_{1}, y_{1}, z_{1}\right)$ adalah $\frac{\partial f\left(x_{1}, y_{1}, z_{1}\right)}{\partial x_{1}}=$ $0, \frac{\partial f\left(x_{1}, y_{1}, z_{1}\right)}{\partial y_{1}}=0, \operatorname{dan} \frac{\partial f\left(x_{1}, y_{1}, z_{1}\right)}{\partial z_{1}}=0$.

Penggolongan konikoida dapat ditentukan menurut pusat konikoida dengan menyelidiki rank matriks:

$A=\left[\begin{array}{lll}a_{11} & a_{12} & a_{13} \\ a_{12} & a_{22} & a_{23} \\ a_{13} & a_{23} & a_{33}\end{array}\right]$ dan $[A, b]=\left[\begin{array}{llll}a_{11} & a_{12} & a_{13} & a_{14} \\ a_{12} & a_{22} & a_{23} & a_{24} \\ a_{13} & a_{23} & a_{33} & a_{34}\end{array}\right]$

Teorema 2.3. Misalkan persamaan konikoida $f(x, y, z)=a_{11} x^{2}+a_{22} y^{2}+a_{33} z^{2}+2 a_{12} x y+$ $2 a_{13} x z+2 a_{23} y z+2 a_{14} x+2 a_{24} y+2 a_{34} z+a_{44}=0$ dengan rank matriks:

$$
A=\left[\begin{array}{lll}
a_{11} & a_{12} & a_{13} \\
a_{12} & a_{22} & a_{23} \\
a_{13} & a_{23} & a_{33}
\end{array}\right] \text { dan }[A, b]=\left[\begin{array}{llll}
a_{11} & a_{12} & a_{13} & a_{14} \\
a_{12} & a_{22} & a_{23} & a_{24} \\
a_{13} & a_{23} & a_{33} & a_{34}
\end{array}\right]
$$

Maka penggolongan konikoida ditentukan dengan cara:

(1) Apabila rank matriks $A=\operatorname{rank}$ matriks $[A, b]=3$. Diperoleh satu titik pusat yang merupakan salah satu dari: Elipsoida (nyata / khayal), hiperboloida daun satu, hiperboloida daun dua. Pusat konikoida $P\left(x_{1}, y_{1}, z_{1}\right)$, jika $f\left(x_{1}, y_{1}, z_{1}\right) \neq 0$ (Bukan merupakan kerucut) dan jika $f\left(x_{1}, y_{1}, z_{1}\right)=0$ (merupakan kerucut (nyata/khayal)).

(2) Apabila rank matriks $\mathrm{A}=2$ sedangkan rank matriks $[A, b]=3$.

Tidak diperoleh titik pusat (titik pusat di tak hingga) yang terdapat pada paraboloida eliptik dan paraboloida hiperbolik.

(3) Apabila rank matriks $A=\operatorname{rank}$ matriks $[A, b]=2$. Tempat kedudukan titik pusat berupa garis lurus yang terdapat pada: Silinder eliptik (nyata/khayal), silinder hiperbolik atau sepasang bidang rata berpotongan (nyata/khayal).

(4) Apabila rank matriks $A=1$ sedangkan rank matriks $[A, b] \neq 1$. Tempat kedudukan titik pusat berupa garis lurus di tak berhingga yang terdapat pada silinder parabolik.

(5) Bila rank matriks $A=\operatorname{rank}$ matriks $[A, b]=1$. Tempat kedudukan titik pusat berupa bidang rata, yang terdapat pada sepasang bidang rata sejajar atau sepasang bidang rata berimpit (nyata/khayal).

Bukti (2.3.1)

a. Ambil $a_{11}=p, a_{22}=q, a_{33}=r, a_{44}=-t$ dan $a_{12}=a_{13}=a_{23}=a_{24}=a_{14}=a_{34}=0$ dengan $p, q, r, t \in$ bilangan positif, maka:

$$
\begin{aligned}
& A=\left[\begin{array}{lll}
a_{11} & a_{12} & a_{13} \\
a_{12} & a_{22} & a_{23} \\
a_{13} & a_{23} & a_{33}
\end{array}\right]=\left[\begin{array}{lll}
p & 0 & 0 \\
0 & q & 0 \\
0 & 0 & r
\end{array}\right] \Rightarrow \operatorname{Rank} A=3 \\
& {[A, b]=\left[\begin{array}{llll}
a_{11} & a_{12} & a_{13} & a_{14} \\
a_{12} & a_{22} & a_{23} & a_{24} \\
a_{13} & a_{23} & a_{33} & a_{34}
\end{array}\right]=\left[\begin{array}{llll}
p & 0 & 0 & 0 \\
0 & q & 0 & 0 \\
0 & 0 & r & 0
\end{array}\right] \Rightarrow \operatorname{Rank}[A, b]=3}
\end{aligned}
$$

Karena $\operatorname{rank} A=\operatorname{rank}[A, b]=3$, akibatnya diperoleh: 


$$
\begin{aligned}
& f(x, y, z)=a_{11} x^{2}+a_{22} y^{2}+a_{33} z^{2}+2 a_{12} x y+2 a_{13} x z+2 a_{23} y z+2 a_{14} x+ \\
& \quad 2 a_{24} y+2 a_{34} z+a_{44}=0 . \\
& \Rightarrow f(x, y, z)=p x^{2}+q y^{2}+r z^{2}-t=0 . \\
& \Rightarrow p x^{2}+q y^{2}+r z^{2}-t=0 . \\
& \Rightarrow p x^{2}+q y^{2}+r z^{2}-t=0 . \\
& \Rightarrow p x^{2}+q y^{2}+r z^{2}=t . \\
& \Rightarrow \frac{p}{t} x^{2}+\frac{q}{t} y^{2}+\frac{r}{t} z^{2}=1 . \\
& \Rightarrow \frac{x^{2}}{\frac{t}{p}}+\frac{y^{2}}{\frac{t}{q}}+\frac{z^{2}}{\frac{t}{r}}=1 . \\
& \Rightarrow \frac{x^{2}}{\left(\sqrt{\frac{t}{p}}\right)^{2}}+\frac{y^{2}}{\left(\sqrt{\frac{t}{q}}\right)^{2}}+\frac{z^{2}}{\left(\sqrt{\frac{t}{r}}\right)^{2}}=1 \text { (merupakan elipsoida nyata) }
\end{aligned}
$$

Jika diambil $a_{44}=t$ maka:

$$
\begin{aligned}
& f(x, y, z)=p x^{2}+q y^{2}+r z^{2}+t=0 . \\
\Rightarrow & p x^{2}+q y^{2}+r z^{2}+t=0 . \\
\Rightarrow & p x^{2}+q y^{2}+r z^{2}=-t . \\
\Rightarrow & \frac{p}{t} x^{2}+\frac{q}{t} y^{2}+\frac{r}{t} z^{2}=-1 . \\
\Rightarrow & \frac{x^{2}}{\frac{t}{p}}+\frac{y^{2}}{\frac{t}{q}}+\frac{z^{2}}{\frac{t}{r}}=-1 . \\
\Rightarrow & \frac{x^{2}}{\left(\sqrt{\frac{t}{p}}\right)^{2}}+\frac{y^{2}}{\left(\sqrt{\frac{t}{q}}\right)^{2}}+\frac{z^{2}}{\left(\sqrt{\frac{t}{r}}\right)^{2}}=-1 \text { (merupakan elipsoida khayal). }
\end{aligned}
$$

b. Ambil $a_{11}=p, a_{22}=q, a_{33}=-r, a_{44}=-t$ dan $a_{12}=a_{13}=a_{23}=a_{24}=a_{14}=a_{34}=0$ dengan $p, q, r, t \in$ bilangan positif, maka:

$$
\begin{aligned}
& A=\left[\begin{array}{lll}
a_{11} & a_{12} & a_{13} \\
a_{12} & a_{22} & a_{23} \\
a_{13} & a_{23} & a_{33}
\end{array}\right]=\left[\begin{array}{lll}
p & 0 & 0 \\
0 & q & 0 \\
0 & 0 & -r
\end{array}\right] \Rightarrow \quad \operatorname{Rank} A=3 \\
& {[A, b]=\left[\begin{array}{llll}
a_{11} & a_{12} & a_{13} & a_{14} \\
a_{12} & a_{22} & a_{23} & a_{24} \\
a_{13} & a_{23} & a_{33} & a_{34}
\end{array}\right]=\left[\begin{array}{cccc}
p & 0 & 0 & 0 \\
0 & q & 0 & 0 \\
0 & 0 & -r & 0
\end{array}\right] \quad \operatorname{Rank}[A, b]=3}
\end{aligned}
$$

Karena $\operatorname{rank} \mathrm{A}=\operatorname{rank}[\mathrm{A}, \mathrm{b}]=3$, akibatnya diperoleh:

$$
\begin{aligned}
& f(x, y, z)=a_{11} x^{2}+a_{22} y^{2}+a_{33} z^{2}+2 a_{12} x y+2 a_{13} x z+2 a_{23} y z+2 a_{14} x+ \\
& \quad 2 a_{24} y+2 a_{34} z+a_{44}=0 . \\
& \Rightarrow f(x, y, z)=p x^{2}+q y^{2}-r z^{2}-t=0 . \\
& \Rightarrow p x^{2}+q y^{2}-r z^{2}-t=0 . \\
& \Rightarrow p x^{2}+q y^{2}-r z^{2}-t=0 . \\
& \Rightarrow p x^{2}+q y^{2}-r z^{2}=t . \\
& \Rightarrow \frac{p}{t} x^{2}+\frac{q}{t} y^{2}-\frac{r}{t} z^{2}=1 . \\
& \Rightarrow \frac{x^{2}}{\frac{t}{p}}+\frac{y^{2}}{\frac{t}{q}}-\frac{z^{2}}{\frac{t}{r}}=1 . \\
& \Rightarrow \frac{x^{2}}{\left(\sqrt{\frac{t}{p}}\right)^{2}}+\frac{y^{2}}{\left(\sqrt{\frac{t}{q}}\right)^{2}}-\frac{z^{2}}{\left(\sqrt{\frac{t}{r}}\right)^{2}}=1 \text { (merupakan hiperboloida daun satu) }
\end{aligned}
$$

Jika diambil $a_{22}=-q$ maka:

$$
f(x, y, z)=p x^{2}-q y^{2}-r z^{2}-t=0 .
$$

$\Rightarrow p x^{2}-q y^{2}-r z^{2}-t=0$.

$\Rightarrow p x^{2}-q y^{2}-r z^{2}=t$.

$\Rightarrow \frac{p}{t} x^{2}-\frac{q}{t} y^{2}-\frac{r}{t} z^{2}=1$.

$\Rightarrow \frac{x^{2}}{\frac{t}{p}}-\frac{y^{2}}{\frac{t}{q}}-\frac{z^{2}}{\frac{t}{r}}=1$.

$\Rightarrow \frac{x^{2}}{\left(\sqrt{\frac{t}{p}}\right)^{2}}-\frac{y^{2}}{\left(\sqrt{\frac{t}{q}}\right)^{2}}-\frac{z^{2}}{\left(\sqrt{\frac{t}{r}}\right)^{2}}=1$ (merupakan hiperboloida daun dua). 
c. Ambil $a_{11}=p, a_{22}=q, a_{33}=-r, a_{44}=-t$ dan $a_{12}=a_{13}=a_{23}=a_{24}=a_{14}=a_{34}=0$ dengan $p, q, r, t \in$ bilangan positif, maka:

$A=\left[\begin{array}{lll}a_{11} & a_{12} & a_{13} \\ a_{12} & a_{22} & a_{23} \\ a_{13} & a_{23} & a_{33}\end{array}\right]=\left[\begin{array}{ccc}p & 0 & 0 \\ 0 & q & 0 \\ 0 & 0 & -r\end{array}\right] \Rightarrow \operatorname{Rank} A=3$

$[A, b]=\left[\begin{array}{llll}a_{11} & a_{12} & a_{13} & a_{14} \\ a_{12} & a_{22} & a_{23} & a_{24} \\ a_{13} & a_{23} & a_{33} & a_{34}\end{array}\right]=\left[\begin{array}{cccc}p & 0 & 0 & 0 \\ 0 & q & 0 & 0 \\ 0 & 0 & -r & 0\end{array}\right] \Rightarrow \operatorname{Rank}[A, b]=3$

Karena $\operatorname{rank} A=\operatorname{rank}[A, b]=3$, akibatnya diperoleh:

$f(x, y, z)=a_{11} x^{2}+a_{22} y^{2}+a_{33} z^{2}+2 a_{12} x y+2 a_{13} x z+2 a_{23} y z+2 a_{14} x+$ $2 a_{24} y+2 a_{34} z+a_{44}=0$.

$\Rightarrow f(x, y, z)=p x^{2}+q y^{2}-r z^{2}-t=0$.

Pusat $P\left(x_{1}, y_{1}, z_{1}\right)$ maka $f\left(x_{1}, y_{1}, z_{1}\right)=p x_{1}{ }^{2}+q y_{1}{ }^{2}-r z_{1}{ }^{2}-t=0$, karena:

$\frac{\partial f\left(x_{1}, y_{1}, z_{1}\right)}{\partial x_{1}}=0 \Rightarrow 2 p x_{1}=0 \Rightarrow x_{1}=0$

$\frac{\partial f\left(x_{1}, y_{1}, z_{1}\right)}{\partial y_{1}}=0 \Rightarrow 2 q y_{1}=0 \quad \Rightarrow y_{1}=0$

$\frac{\partial f\left(x_{1}, y_{1}, z_{1}\right)}{\partial z_{1}}=0 \Rightarrow-2 r z_{1}=0 \Rightarrow z_{1}=0$

Diperoleh Pusat $P\left(x_{1}, y_{1}, z_{1}\right)=P(0,0,0)$ sehingga:

$f\left(x_{1}, y_{1}, z_{1}\right)=f(0,0,0)=-t \neq 0$

Karena:

$f(x, y, z)=p x^{2}+q y^{2}-r z^{2}-t=0$

$\Longrightarrow p x^{2}+q y^{2}-r z^{2}-t=0$.

$\Rightarrow p x^{2}+q y^{2}-r z^{2}-t=0$.

$\Rightarrow p x^{2}+q y^{2}-r z^{2}=t$.

$\Rightarrow \frac{p}{t} x^{2}+\frac{q}{t} y^{2}-\frac{r}{t} z^{2}=1$.

$\Rightarrow \frac{x^{2}}{\frac{t}{p}}+\frac{y^{2}}{\frac{t}{q}}-\frac{z^{2}}{\frac{t}{r}}=1$.

$\Longrightarrow \frac{x^{2}}{\left(\sqrt{\frac{t}{p}}\right)^{2}}+\frac{y^{2}}{\left(\sqrt{\frac{t}{q}}\right)^{2}}-\frac{z^{2}}{\left(\sqrt{\frac{t}{r}}\right)^{2}}=1$ (merupakan hiperboloida daun satu / bukan merupakan kerucut).

d. Ambil $a_{11}=p, a_{22}=q, a_{33}=-r$, dan $a_{12}=a_{13}=a_{23}=a_{24}=a_{14}=a_{34}=a_{44}=0$ dengan $p, q, r \in$ bilangan positif, maka:

$$
\begin{aligned}
& A=\left[\begin{array}{lll}
a_{11} & a_{12} & a_{13} \\
a_{12} & a_{22} & a_{23} \\
a_{13} & a_{23} & a_{33}
\end{array}\right]=\left[\begin{array}{lll}
p & 0 & 0 \\
0 & q & 0 \\
0 & 0 & -r
\end{array}\right] \Rightarrow \operatorname{Rank} A=3 \\
& {[A, b]=\left[\begin{array}{llll}
a_{11} & a_{12} & a_{13} & a_{14} \\
a_{12} & a_{22} & a_{23} & a_{24} \\
a_{13} & a_{23} & a_{33} & a_{34}
\end{array}\right]=\left[\begin{array}{cccc}
p & 0 & 0 & 0 \\
0 & q & 0 & 0 \\
0 & 0 & -r & 0
\end{array}\right] \Rightarrow \operatorname{Rank}[A, b]=3}
\end{aligned}
$$

Karena $\operatorname{rank} A=\operatorname{rank}[A, b]=3$, akibatnya diperoleh:

$$
\begin{aligned}
& f(x, y, z)=a_{11} x^{2}+a_{22} y^{2}+a_{33} z^{2}+2 a_{12} x y+2 a_{13} x z+2 a_{23} y z+2 a_{14} x+ \\
& 2 a_{24} y+2 a_{34} z+a_{44}=0 \text {. } \\
& \Rightarrow f(x, y, z)=p x^{2}+q y^{2}-r z^{2}=0 \text {. }
\end{aligned}
$$

Pusat $P\left(x_{1}, y_{1}, z_{1}\right)$ maka $f\left(x_{1}, y_{1}, z_{1}\right)=p x_{1}^{2}+q y_{1}{ }^{2}-r z_{1}{ }^{2}=0$, karena:

$\frac{\partial f\left(x_{1}, y_{1}, z_{1}\right)}{\partial x_{1}}=0 \Longrightarrow 2 p x_{1}=0 \Longrightarrow x_{1}=0$
$\frac{\partial f\left(x_{1}, y_{1}, z_{1}\right)}{\partial y_{1}}=0 \Longrightarrow 2 q y_{1}=0 \Rightarrow y_{1}=0$
$\frac{\partial f\left(x_{1}, y_{1}, z_{1}\right)}{\partial z_{1}}=0 \Longrightarrow-2 r z_{1}=0 \Longrightarrow z_{1}=0$

Diperoleh Pusat $P\left(x_{1}, y_{1}, z_{1}\right)=P(0,0,0)$ sehingga:

$f\left(x_{1}, y_{1}, z_{1}\right)=f(0,0,0)=0$

Karena:

$f(x, y, z)=p x^{2}+q y^{2}-r z^{2}=0$

$\Rightarrow p x^{2}+q y^{2}-r z^{2}=0$. 


$$
\begin{aligned}
& \Rightarrow p x^{2}+q y^{2}-r z^{2}=0 \\
& \Rightarrow p x^{2}+q y^{2}-r z^{2}=0 \\
& \Rightarrow \frac{x^{2}}{\frac{1}{p}}+\frac{y^{2}}{\frac{1}{q}}-\frac{z^{2}}{\frac{1}{r}}=0 \\
& \Rightarrow \frac{x^{2}}{\left(\sqrt{\frac{1}{p}}\right)^{2}}+\frac{y^{2}}{\left(\sqrt{\frac{1}{q}}\right)^{2}}-\frac{z^{2}}{\left(\sqrt{\frac{1}{r}}\right)^{2}}=0 \text { (merupakan kerucut nyata). }
\end{aligned}
$$

Jika diambil $a_{33}=r$, maka:

$$
\begin{aligned}
& f(x, y, z)=p x^{2}+q y^{2}+r z^{2}=0 \\
& \Rightarrow p x^{2}+q y^{2}+r z^{2}=0 . \\
& \Rightarrow p x^{2}+q y^{2}+r z^{2}=0 . \\
& \Rightarrow p x^{2}+q y^{2}+r z^{2}=0 \\
& \Rightarrow \frac{x^{2}}{\frac{1}{p}}+\frac{y^{2}}{\frac{1}{q}}+\frac{z^{2}}{\frac{1}{r}}=0 . \\
& \Rightarrow \frac{x^{2}}{\left(\sqrt{\frac{1}{p}}\right)^{2}}+\frac{y^{2}}{\left(\sqrt{\frac{1}{q}}\right)^{2}}+\frac{z^{2}}{\left(\sqrt{\frac{1}{r}}\right)^{2}}=0 \text { (merupakan kerucut khayal). }
\end{aligned}
$$

\section{Bukti (2.3.2)}

Ambil $a_{11}=p, a_{22}=q, a_{34}=-r$ dan $a_{12}=a_{13}=a_{23}=a_{24}=a_{14}=a_{33}=a_{44}=0$ dengan $p, q \in$ bilangan positif, maka:

$A=\left[\begin{array}{lll}a_{11} & a_{12} & a_{13} \\ a_{12} & a_{22} & a_{23} \\ a_{13} & a_{23} & a_{33}\end{array}\right]=\left[\begin{array}{lll}p & 0 & 0 \\ 0 & q & 0 \\ 0 & 0 & 0\end{array}\right] \Rightarrow \operatorname{Rank} A=2$
$[A, b]=\left[\begin{array}{llll}a_{11} & a_{12} & a_{13} & a_{14} \\ a_{12} & a_{22} & a_{23} & a_{24} \\ a_{13} & a_{23} & a_{33} & a_{34}\end{array}\right]=\left[\begin{array}{cccc}p & 0 & 0 & 0 \\ 0 & q & 0 & 0 \\ 0 & 0 & 0 & -r\end{array}\right] \Rightarrow \operatorname{Rank}[\mathrm{A}, \mathrm{b}]=3$

Karena rank $A=2$ dan rank $[A, b]=3$, akibatnya diperoleh:

$f(x, y, z)=a_{11} x^{2}+a_{22} y^{2}+a_{33} z^{2}+2 a_{12} x y+2 a_{13} x z+2 a_{23} y z+2 a_{14} x+$
$2 a_{24} y+2 a_{34} z+a_{44}=0$.

$\Rightarrow f(x, y, z)=p x^{2}+q y^{2}-2 r z=0$.

$\Rightarrow p x^{2}+q y^{2}=2 r z$.

$\Rightarrow \frac{x^{2}}{\frac{1}{p}}+\frac{y^{2}}{\frac{1}{q}}=\frac{2 z}{\frac{1}{r}}$.

$\Rightarrow \frac{x^{2}}{\left(\sqrt{\frac{1}{p}}\right)^{2}}+\frac{y^{2}}{\left(\sqrt{\frac{1}{q}}\right)^{2}}=\frac{2 z}{\frac{1}{r}}$ (merupakan paraboloida eliptik).

Jika diambil $a_{22}=-q$, maka:

$f(x, y, z)=p x^{2}-q y^{2}-2 r z=0$.

$\Rightarrow p x^{2}-q y^{2}=2 r z$.

$\Rightarrow \frac{x^{2}}{\frac{1}{p}}-\frac{y^{2}}{\frac{1}{q}}=\frac{2 z}{\frac{1}{r}}$.

$\Rightarrow \frac{x^{2}}{\left(\sqrt{\frac{1}{p}}\right)^{2}}-\frac{y^{2}}{\left(\sqrt{\frac{1}{q}}\right)^{2}}=\frac{2 z}{\frac{1}{r}}$ (merupakan paraboloida hiperbolik)

\section{Bukti (2.3.3)}

a. Ambil $a_{11}=p, \quad a_{22}=q, \quad a_{44}=-r$ dan $a_{12}=a_{13}=a_{23}=a_{24}=a_{14}=a_{33}=a_{34}=0$ dengan $p, q, r \in$ bilangan positif, maka:

$$
\begin{aligned}
& A=\left[\begin{array}{lll}
a_{11} & a_{12} & a_{13} \\
a_{12} & a_{22} & a_{23} \\
a_{13} & a_{23} & a_{33}
\end{array}\right]=\left[\begin{array}{lll}
p & 0 & 0 \\
0 & q & 0 \\
0 & 0 & 0
\end{array}\right] \Rightarrow \operatorname{Rank} \mathrm{A}=2 \\
& {[A, b]=\left[\begin{array}{lllll}
a_{11} & a_{12} & a_{13} & a_{14} \\
a_{12} & a_{22} & a_{23} & a_{24} \\
a_{13} & a_{23} & a_{33} & a_{34}
\end{array}\right]=\left[\begin{array}{llll}
p & 0 & 0 & 0 \\
0 & q & 0 & 0 \\
0 & 0 & 0 & 0
\end{array}\right] \Rightarrow \operatorname{Rank}[\mathrm{A}, \mathrm{b}]=2}
\end{aligned}
$$

Karena rank $\mathrm{A}=$ rank $[\mathrm{A}, \mathrm{b}]=2$, akibatnya diperoleh: 


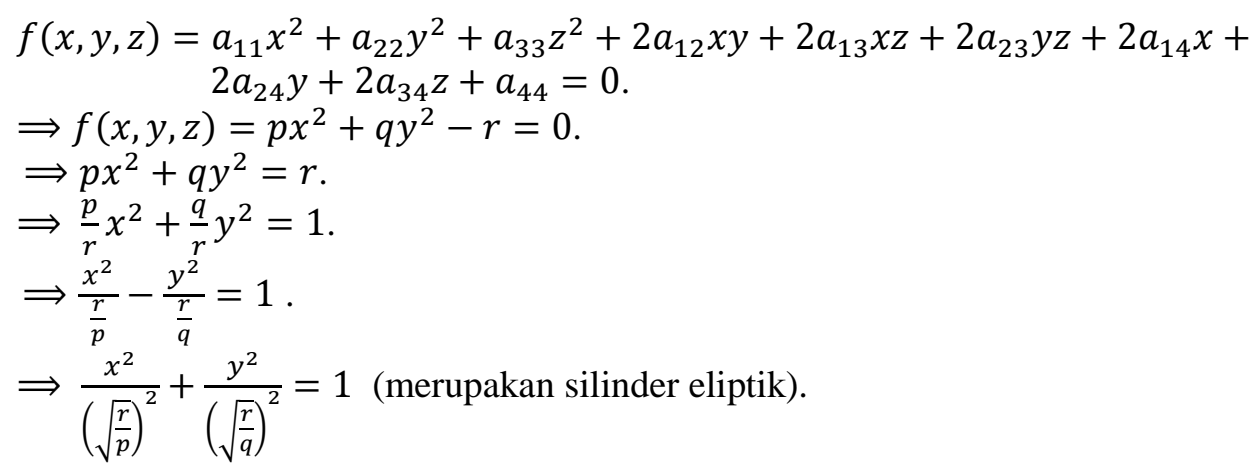

Jika diambil $a_{22}=-q$, diperoleh:

$f(x, y, z)=p x^{2}-q y^{2}-r=0$.

$\Rightarrow p x^{2}-q y^{2}=r$.

$\Rightarrow \frac{p}{r} x^{2}-\frac{q}{r} y^{2}=1$.

$\Rightarrow \frac{x^{2}}{\frac{r}{p}}-\frac{y^{2}}{\frac{r}{q}}=1$.

$\Rightarrow \frac{x^{2}}{\left(\sqrt{\frac{r}{p}}\right)^{2}}-\frac{y^{2}}{\left(\sqrt{\frac{r}{q}}\right)^{2}}=1$ (merupakan silinder hiperbolik).

b. Ambil $a_{11}=p, a_{22}=-p \quad$ dan $\quad a_{12}=a_{13}=a_{14}=a_{23}=a_{24}=a_{33}=a_{34}=a_{44}=0$ dengan $p \in$ bilangan positif, maka:

$$
\begin{aligned}
& A=\left[\begin{array}{lll}
a_{11} & a_{12} & a_{13} \\
a_{12} & a_{22} & a_{23} \\
a_{13} & a_{23} & a_{33}
\end{array}\right]=\left[\begin{array}{ccc}
p & 0 & 0 \\
0 & -p & 0 \\
0 & 0 & 0
\end{array}\right] \Rightarrow \operatorname{Rank} A=2 \\
& {[A, b]=\left[\begin{array}{llll}
a_{11} & a_{12} & a_{13} & a_{14} \\
a_{12} & a_{22} & a_{23} & a_{24} \\
a_{13} & a_{23} & a_{33} & a_{34}
\end{array}\right]=\left[\begin{array}{cccc}
p & 0 & 0 & 0 \\
0 & -p & 0 & 0 \\
0 & 0 & 0 & 0
\end{array}\right] \Rightarrow \operatorname{Rank}[A, b]=2}
\end{aligned}
$$

Karena $\operatorname{rank} A=\operatorname{rank}[A, b]=2$, akibatnya diperoleh:

$f(x, y, z)=a_{11} x^{2}+a_{22} y^{2}+a_{33} z^{2}+2 a_{12} x y+2 a_{13} x z+2 a_{23} y z+2 a_{14} x+2 a_{24} y+$ $2 a_{34} z+a_{44}=0$

$f(x, y, z)=p x^{2}-p y^{2}=0$.

$\Longrightarrow p\left(x^{2}-y^{2}\right)=0$.

$\Rightarrow x^{2}-y^{2}=0$.

$\Longrightarrow(x+y)(x-y)=0$ (merupakan sepasang bidang rata nyata berpotongan).

Jika diambil $a_{22}=0$ dan $a_{44}=1$, maka:

$f(x, y, z)=p x^{2}+1=0$.

$\Rightarrow p\left(x^{2}+1\right)=0$.

$\Rightarrow x^{2}+1=0$.

$\Rightarrow x^{2}=-1$.

$\Rightarrow x= \pm \sqrt{-1}$ (merupakan sepasang bidang rata khayal berpotongan).

\section{Bukti (2.3.4)}

Ambil $a_{22}=a_{14}=p$ dan $a_{11}=a_{12}=a_{13}=a_{23}=a_{24}=a_{34}=a_{33}=a_{44}=0$ dengan $p \in$ bilangan positif, maka:

$A=\left[\begin{array}{lll}a_{11} & a_{12} & a_{13} \\ a_{12} & a_{22} & a_{23} \\ a_{13} & a_{23} & a_{33}\end{array}\right]=\left[\begin{array}{lll}0 & 0 & 0 \\ 0 & p & 0 \\ 0 & 0 & 0\end{array}\right] \Rightarrow \operatorname{Rank} A=1$

$[A, b]=\left[\begin{array}{llll}a_{11} & a_{12} & a_{13} & a_{14} \\ a_{12} & a_{22} & a_{23} & a_{24} \\ a_{13} & a_{23} & a_{33} & a_{34}\end{array}\right]=\left[\begin{array}{llll}0 & 0 & 0 & p \\ 0 & p & 0 & 0 \\ 0 & 0 & 0 & 0\end{array}\right] \Rightarrow \operatorname{Rank}[A, b]=2 \neq 1$

Karena $\operatorname{rank} A=1$ dan rank $[A, b] \neq 1$, akibatnya diperoleh:

$f(x, y, z)=a_{11} x^{2}+a_{22} y^{2}+a_{33} z^{2}+2 a_{12} x y+2 a_{13} x z+2 a_{23} y z+2 a_{14} x+$

$$
2 a_{24} y+2 a_{34} z+a_{44}=0 \text {. }
$$

$\Rightarrow f(x, y, z)=p y^{2}-2 p x=0$.

$\Rightarrow p y^{2}-2 p x=0$. 
$\Longrightarrow p\left(y^{2}-2 x\right)=0$.

$\Rightarrow y^{2}-2 x=0$.

$\Rightarrow y^{2}=2 x$ (merupakan silinder parabolik).

Bukti (2.3.5)

Ambil $a_{11}=a_{44}=p, a_{14}=-p$ dan $a_{11}=a_{12}=a_{13}=a_{23}=a_{24}=a_{34}=a_{33}=0$ dengan $p \in$ bilangan positif, maka:

$A=\left[\begin{array}{lll}a_{11} & a_{12} & a_{13} \\ a_{12} & a_{22} & a_{23} \\ a_{13} & a_{23} & a_{33}\end{array}\right]=\left[\begin{array}{ccc}p & 0 & 0 \\ 0 & 0 & 0 \\ 0 & 0 & 0\end{array}\right] \Rightarrow \operatorname{Rank} A=1$

$[A, b]=\left[\begin{array}{llll}a_{11} & a_{12} & a_{13} & a_{14} \\ a_{12} & a_{22} & a_{23} & a_{24} \\ a_{13} & a_{23} & a_{33} & a_{34}\end{array}\right]=\left[\begin{array}{cccc}p & 0 & 0 & -p \\ 0 & 0 & 0 & 0 \\ 0 & 0 & 0 & 0\end{array}\right] \Rightarrow \operatorname{Rank}[A, b]=1$

Karena $\operatorname{rank} A=1$ dan $\operatorname{rank}[A, b]=1$, akibatnya diperoleh:

$f(x, y, z)=a_{11} x^{2}+a_{22} y^{2}+a_{33} z^{2}+2 a_{12} x y+2 a_{13} x z+2 a_{23} y z+2 a_{14} x+2 a_{24} y+2 a_{34} z+$ $a_{44}=0$.

$f(x, y, z)=p x^{2}-2 p x+p=0$.

$\Rightarrow p x^{2}-2 p x+p=0$.

$\Rightarrow p\left(x^{2}-2 x+1\right)=0$.

$\Rightarrow x^{2}-2 x+1=0$.

$\Rightarrow(x-1)^{2}=0$

$\Longrightarrow(x-1)(x-1)=0$ (merupakan sepasang bidang rata nyata berhimpit).

Jika diambil $a_{14}=0$, maka diperoleh:

$$
f(x, y, z)=p x^{2}+p=0 .
$$

$\Rightarrow p\left(x^{2}+1\right)=0$.

$\Rightarrow\left(x^{2}+1\right)=0$.

$\Rightarrow\left(x^{2}+1\right)^{2}=0$.

$\Rightarrow\left(x^{2}+1\right)\left(x^{2}+1\right)=0$. (merupakan sepasang bidang rata khayal berhimpit).

\section{Kesimpulan}

Mengacu pada pembahasan mengenai penggolongan konikoida berdasarkan kerucut arahnya (KA) yaitu:

1. KA nyata dan tidak berubah corak maka konikoidanya salah satu dari hiperboloida daun satu atau hiperboloida daun dua.

2. KA khayal dan tidak berubah corak maka konikoidanya salah satu dari elipsoida atau kerucut khayal.

3. KA berubah corak menjadi sepasang bidang rata nyata berpotongan maka konikoidanya salah satu dari paraboloida hiperbolik atau silinder.

4. KA berubah corak menjadi sepasang bidang rata khayal maka konikoidanya merupakan salah satu paraboloida eliptik atau silinder eliptik.

5. KA berubah corak menjadi sepasang bidang rata berhimpit maka konikoidanya merupakan silinder parabolik atau sepasang bidang rata sejajar.

Dan penggolongan konikoida menurut pusat konikoida yaitu dengan menyelidiki rank matriks:

$A=\left[\begin{array}{lll}a_{11} & a_{12} & a_{13} \\ a_{12} & a_{22} & a_{23} \\ a_{13} & a_{23} & a_{33}\end{array}\right]$ dan $[A, b]=\left[\begin{array}{llll}a_{11} & a_{12} & a_{13} & a_{14} \\ a_{12} & a_{22} & a_{23} & a_{24} \\ a_{13} & a_{23} & a_{33} & a_{34}\end{array}\right]$

(1) Apabila rank matriks $A=$ rank matriks $[A, b]=3$. Diperoleh satu titik pusat yang merupakan salah satu dari: Elipsoida (nyata / khayal), hiperboloida daun satu, hiperboloida daun dua. Pusat konikoida $P\left(x_{1}, y_{1}, z_{1}\right)$, jika $f\left(x_{1}, y_{1}, z_{1}\right) \neq 0$ (Bukan merupakan kerucut) dan jika $f\left(x_{1}, y_{1}, z_{1}\right)=0$ (merupakan kerucut (nyata/khayal)).

(2) Apabila rank matriks $A=2$ sedangkan rank matriks $[A, b]=3$. Tidak diperoleh titik pusat (titik pusat di tak hingga) yang terdapat pada paraboloida eliptik dan paraboloida hiperbolik.

(3) Apabila rank matriks $A=\operatorname{rank}$ matriks $[A, b]=2$. Tempat kedudukan titik pusat berupa garis lurus.yang terdapat pada : Silinder eliptik (nyata/khayal), silinder hiperbolik atau sepasang bidang rata berpotongan (nyata/khayal). 
(4) Apabila rank matriks $A=1$ sedangkan rank matriks $[A, b] \neq 1$. Tempat kedudukan titik pusat berupa garis lurus di tak berhingga yang terdapat pada silinder parabolik.

(5) Bila rank matriks $A=$ rank matriks $[A, b]=1$. Tempat kedudukan titik pusat berupa bidang rata, yang terdapat pada sepasang bidang rata sejajar atau sepasang bidang rata berimpit (nyata/khayal).

Untuk menyelidiki konikoida $f(x, y, z)=a_{11} x^{2}+a_{22} y^{2}+a_{33} z^{2}+2 a_{12} x y+2 a_{13} x z+$ $2 a_{23} y z+2 a_{14} x+2 a_{24} y+2 a_{34} z+a_{44}=0$ dapat dilakukan sebagai berikut:

1. Golongkan terlebih dahulu berdasarkan keadaan titik pusatnya dan rank matriksnya, salah satu dari golongan (1), (2), (3), (4) atau (5).

2. Bila termasuk golongan (1):

a. Apabila pusatnya terletak pada konikoida maka merupakan kerucut.

b. Apabila pusatnya tidak terletak pada konikoida maka diselidiki kerucut arahnya (KA) yaitu:

1) Jika kerucut arahnya khayal maka konikoida merupakan elipsoida.

2) Jika kerucut arahnya nyata maka konikoida merupakan salah satu dari hiperboloida daun satu atau hiperboloida daun dua. Cara membedakan apakah hiperboloida daun satu atau hiperboloida daun dua yaitu dengan cara:

a) Pilih sebarang titik pada hiperboloida.

b) Buat bidang singgung dititik tersebut.

c) Tentukan proyeksi garis potong hiperboloida dan bidang singgung tersebut (yang tidak tegak lurus bidang singgung).

d) Jika proyeksi tersebut nyata maka merupakan hiperboloida daun satu dan jika proyeksinya khayal maka merupakan hiperbolida daun dua.

3. Bila termasuk golongan (2) : Selidiki kerucut arahnya (KA)

a. Apabila kerucut arah (KA) berubah corak menjadi sepasang bidang rata nyata yang berpotongan maka konikoida merupakan parabolida hiperbolik.

b. Apabila kerucut arah (KA) berubah corak menjadi sepasang bidang rata khayal maka konikoda merupakan paraboloida eliptik.

4. Bila termasuk golongan (3): Lakukan pengirisan dengan salah satu bidang koordinat yang tidak sejajar dengan garis / bidang tempat kedudukan (TK) titik pusat.

a. Apabila irisannya elips maka konikoida adalah silinder eliptik (jika elips khayal maka selinder eliptik tersebut khayal).

b. Apabila irisannya hiperbola maka konikoida merupakan silinder hiperbolik.

c. Apabila irisannya berubah corak menjadi sepasang garis lurus berpotongan maka konikoida merupakan sepasang bidang rata berpotongan.

5. Apabila termasuk golongan (4) maka konikoida merupaka silinder hiperbolik.

6. Apabila termasuk golongan (5): Lakukan pengirisan dengan salah satu bidang koordinat yang tidak sejajar dengan garis / bidang tempat kedudukan (TK) titik pusat.

a. Apabila irisannya sepasang garis lurus sejajar, maka konikoida adalah sepasang bidang rata sejajar.

b. Apabila irisannya sepasang garis lurus berimpit, maka konikoida merupakan sepasang bidang rata berimpit.

\section{Ucapan Terima Kasih}

Ucapan terima kasih kami sampaikan kepada PNBP UNM dan kepada rekan-rekan dosen Jurusan Matemtika FMIPA Universitas Negeri Makassar atas kritikan dan saran-sarannya.

\section{Daftar Pustaka}

[1] Suryadi, D. 1986. Teori dan Soal Ilmu Ukur Analitik Ruang. Ghalia Indonesia. Jakarta.

[2] Kartiman, R. 1985. Matematika Tingkat Tinggi Cetakan Ketiga. PT. Pradnya Paramita. Jakarta.

[3] Supranto. J. 1971. Pengantar Matrix. Erlangga. Jakarta. 